\title{
Qual é o tipo de fototerapia mais comumente indicada no tratamento da psoríase? UVB banda estreita e PUVA: comportamento da prescrição *
}

\author{
What is the most common phototherapy prescription for psoriasis:
}

NB-UVB or PUVA? Prescription behavior

\author{
Ida Duarte ${ }^{1}$ \\ Roberta Buense Bedrikow ${ }^{3}$
}

\author{
José Antonio Jabur da Cunha ${ }^{2}$ \\ Rosana Lazzarini ${ }^{4}$
}

\begin{abstract}
Resumo: FunDAMENTOS: Formas moderada e grave de psoríase requerem fototerapia e/ou medicações sistêmicas. Tanto UVB banda estreita quanto fototerapia UVA com psoralênicos (PUVA) podem ser utilizadas no tratamento dessas formas de psoríase, sendo comprovada a efetividade de ambas as terapias.

OBjetrvos: Avaliar as indicações de dois tipos de fototerapia no tratamento da psoríase refratária à terapia tópica: UVB banda estreita e PUVA.

MÉTODos: Entre janeiro de 2006 e dezembro de 2007, os pacientes encaminhados a dois serviços de fototerapia foram incluídos neste estudo. Dados sobre os casos e tipos de prescrição foram coletados de maneira retrospectiva.

Resultados: Dentre os 67 pacientes estudados, 51 (76\%) foram tratados com UVB banda estreita. As razões para sua indicação foram presença de psoríase em gotas (22\%), presença de finas placas (15\%), uso de drogas fotossensibilizantes (15\%), idade abaixo de 20 anos (9\%), fototipo I (9\%) e doença hepática (6\%). Os 16 (24\%) restantes foram tratados com PUVA. A principal indicação dessa terapia foi gravidade da doença (15\%), seguida de fototipo IV (9\%).

CONCLUSÕES: As prescrições de UVB banda estreita excederam as de PUVA devido ao menor número de contraindicações, menor possibilidade de efeitos colaterais, e ainda por ser uma opção mais prática.

Palavras-chave: Fototerapia; Psoríase; Raios ultravioleta; Terapia PUVA
\end{abstract}

\begin{abstract}
BACKGROUND: Moderate and severe forms of psoriasis require phototherapy and / or systemic medications. Both UVA and UVB can be used to treat cases of moderate and severe psoriasis, and the effectiveness of both has been proven.

OBJECTIVE: to access the prescription behavior relating to two types of phototherapy for treating psoriasis refractory to topical treatment: narrowband UVB (NB-UVB) or psoralen plus UVA phototherapy (PUVA).

METHODS: Between January 2006 and December 2007, patients referred to two phototherapy services were included in this study. Data on the cases and on the type of prescription were collected retrospectively.

RESULTS: Among the 67 studied patients, 51 (76\%) were treated with narrowband UVB. The reasons for the indication were the presence of the guttate type of psoriasis (22\%), the presence of thin plaques (15\%), the use of drugs that affected photosensitivity (15\%), age less than 20 years (9\%), skin type I (9\%), and liver disease (6\%). The remaining 16 (24\%) were treated with PUVA. The main indication for this therapy was the severity of the disease (15\%), followed by the presence of skin type IV (9\%).

ConcLusions: Prescriptions of narrowband UVB exceeded those of PUVA because of fewer contraindications and fewer possible side effects, and because it was a more practical option.

Keywords: Phototherapy; Psoriasis; PUVA therapy; Ultraviolet rays
\end{abstract}

Recebido em 26.02.2009.

Aprovado pelo Conselho Consultivo e aceito para publicação em 04.03 .09

* Trabalho realizado no Departamento de Dermatologia, Santa Casa de São Paulo - São Paulo (SP), Brasil.

Conflito de interesse: Nenhum / Conflict of interest: None

Suporte financeiro: Nenhum / Financial funding: None

Professora Doutora da Faculdade de Medicina da Santa Casa de São Paulo. Responsável pelo setor de alergia e fototerapia da Clínica de Dermatologia, Santa Casa de São Paulo - São Paulo (SP), Brasil.

Especializando do Departamento de Dermatologia, Santa Casa de São Paulo - São Paulo (SP), Brasil.

Médica assistente da Clínica de Dermatologia, Santa Casa de São Paulo - São Paulo (SP), Brasil.

Chefe da Clínica de Dermatologia, Santa Casa de São Paulo - São Paulo (SP), Brasil.

(C)2009 by Anais Brasileiros de Dermatologia 


\section{INTRODUÇÃO}

Psoríase é uma doença crônica, inflamatória e recorrente, com manifestações clínicas e gravidade variáveis. Caracteriza-se principalmente por eritema, infiltração e descamação da pele. Estima-se que $2 \%$ a $3 \%$ da população mundial seja afetada pela doença. . $^{1-3}$

Terapias tópicas costumam ser suficientes no controle da psoríase de intensidade leve; porém, as formas moderada e grave requerem outras opções terapêuticas, como fototerapia e medicações sistêmicas.

A luz UV tem propriedades anti-inflamatória, antiproliferativa e imunossupressora. ${ }^{7,8} \mathrm{~A}$ radiação UV é dividida em UVA ( $400-320 \mathrm{~nm}$ ), capaz de alcançar a epiderme e derme profunda, UVB $(320-290 \mathrm{~nm})$, que alcança somente a epiderme, e UVC $(290-200$ $\mathrm{nm})$, que não chega à superfície terrestre. Os raios UVA são subdivididos em UVA I $(400-340 \mathrm{~nm})$ e UVA II $(340-320 \mathrm{~nm})$, e a faixa de UVB entre 311 e $312 \mathrm{~nm}$ é chamada de UVB banda estreita (UVB "narrow band" - UVB NB). O uso deste tipo de UVB no tratamento da psoríase teve início na década de 80 , quando as primeiras lâmpadas de UVB NB foram desenvolvidas (Philips, Eindhoven, Holanda). ${ }^{7,9}$ Subsequentemente esse método provou ser efetivo no controle da psoríase, utilizando-se de doses suberitemogênicas. ${ }^{10}$ Estudos mostraram que UVB NB pode ser mais efetiva que UVB banda larga no tratamento da psoríase, 9,10 consequentemente a escolha atualmente deve ser feita entre UVA e UVB NB.

Tanto UVB NB quanto PUVA podem ser utilizadas no tratamento de formas moderada e grave da psoríase, e vem sendo comprovada a efetividade de ambas as terapias.6,11,12 Dessa forma a seleção entre uma ou outra modalidade de fototerapia deve basearse em outros fatores além da eficácia, incluindo segurança, resposta prévia ao tratamento, gravidade da psoríase e adesão ao tratamento. ${ }^{6}$

O objetivo deste estudo foi avaliar a frequência com que PUVA e UVB NB são prescritas a pacientes com diagnóstico de psoríase que não apresentaram resposta ao tratamento tópico.

\section{MATERIAIS E MÉTODOS}

Entre janeiro de 2006 e dezembro de 2007, pacientes com psoríase refratária aos tratamentos tópicos foram referidos para dois serviços de fototerapia (um hospital universitário e uma clínica particular, ambos com a mesma equipe médica, mesmo protocolo de tratamento e mesmos equipamentos) e incluídos neste estudo retrospectivo. Todos os pacientes receberam indicação de UVB NB ou PUVA.

Foram excluídos do estudo aqueles que estivessem usando qualquer terapia combinada (tópica e/ou sistêmica), ou qualquer medicação sistêmica para psoríase nos dois meses que antecederam o início da fototerapia.

Dados demográficos foram retrospectivamente coletados das fichas médicas dos pacientes sob fototerapia. Foram coletados ainda dados quanto ao tipo de pele (segundo classificação de Fitzpatrick), ${ }^{13}$ tipo de psoríase (vulgar, em gotas ou eritrodérmica), ${ }^{14}$ gravidade da doença, tipo de fototerapia prescrita (PUVA ou UVB NB) e evolução clínica.

As razões preponderantes na escolha do regime de fototerapia foram registradas em prontuário médico e baseadas na idade do paciente, fototipo cutâneo, gravidade da doença, comorbidades e uso de medicações sistêmicas. Os critérios utilizados nos serviços de fototerapia estudados foram: UVB NB como primeira escolha em indivíduos com menos de 20 anos de idade, nos portadores de psoríase gutata ou em finas placas e nos casos com gravidade leve para moderada. Fototerapia PUVA foi primeira opção nos casos extremos com placas grossas e pele tipo IV ou VI. PUVA foi contraindicada nos pacientes com comprometimento hepático ou em uso de drogas fotossensibilizantes; nesses casos a severidade ou fototipo cutâneo não foram considerados, e indicou-se UVB NB.

Todos os pacientes foram submetidos à fototerapia com duas sessões por semana utilizandose equipamento profissional (Prolumina Fototerapia, São Paulo, Brasil: cabine UVA com 48 lâmpadas Philips Sunlamp 100 W-R ou cabine UVB NB com 42 lâmpadas banda estreita Philips TL 100 W/01).

\section{RESULTADOS}

Sessenta e sete pacientes foram tratados durante o período do estudo: 37 homens $(55,2 \%)$ e 30 mulheres $(44,8 \%)$, com idades que variaram de 12 a 87 anos e média de 39 anos de idade. Seis deles (9\%) foram classificados como pele tipo I de Fitzpatrick, 35 $(52,2 \%)$ como tipo II, $15(22,4 \%)$ como tipo III e 11 $(16,4 \%)$ como tipo IV.

Apenas um paciente (1,5\%) apresentava psoríase eritrodérmica, enquanto 16 (24\%) tinham psoríase em gotas e $50(74,5 \%)$ tinham psoríase vulgar com gravidades variáveis.

Dentre os 67 pacientes estudados, 51 (76\%) foram tratados com UVB NB. As razões para indicação de UVB NB estão indicadas na tabela 1 e distribuíramse da seguinte forma: presença de psoríase em gotas (22\%), presença de finas placas (15\%), uso de drogas que interferem na fotossensibilidade (15\%), idade menor que 20 anos (9\%) fototipo I (9\%) e presença de hepatopatia associada (6\%). Os 16 (24\%) restantes foram tratados com PUVA. A principal indicação desse tipo de terapia (Tabela 2) foi gravidade da doença (10 
TABela 1: Razões determinantes na escolha por UVB NB em 51/67 (76\%) pacientes psoriásicos em dois serviços de fototerapia

\begin{tabular}{lcl}
\hline Razóes para indicação & Número de pacientes & $\begin{array}{l}\text { Porcentagem } \\
\text { (67 pacientes) }\end{array}$ \\
\hline Psoríase em gotas & 15 & 22 \\
Finas Placas & 10 & 15 \\
Uso de drogas que afetam fotossensibilidade & 10 & 15 \\
Idade < 20 anos & 6 & 9 \\
Fototipo I & 6 & 9 \\
Doenças hepáticas & 4 & 6 \\
\hline Total & $\mathbf{5 1}$ & $\mathbf{7 6}$ \\
\hline
\end{tabular}

TABela 2: Razões determinantes na escolha por terapia PUVA em 16/67 (24\%) pacientes psoriásicos em dois serviços de fototerapia

\begin{tabular}{lcc}
\hline Razões para indicação & Número de pacientes & $\begin{array}{c}\text { Porcentagem } \\
\text { (67 pacientes) }\end{array}$ \\
\hline Gravidade da doença & 10 & 15 \\
Fototipo IV & $6^{*}$ & 9 \\
\hline Total & 16 & 24 \\
\hline
\end{tabular}

*Nos casos de comprometimento hepático ou uso de drogas fotossensibilizantes, desconsiderou-se a severidade ou fototipo cutâneo, e indicou-se UVB NB.

pacientes, $15 \%$ ), seguida pela presença de pele tipo IV (6 pacientes, 9\%). A figura 1 ilustra como se deu a prescrição da fototerapia nos pacientes avaliados.

\section{DISCUSSÃO}

Muitos estudos têm comparado a eficácia das terapias UVB NB e PUVA na psoríase moderada a grave. ${ }^{15-17}$ A heterogeneidade considerável entre esses estudos no que diz respeito à gravidade da doença, subtipos de psoríase, fototipo cutâneo, regimes de fototerapia e métodos utilizados na mensuração dos resultados tornaram difícil a condução de uma revisão sistemática consistente. Embora PUVA venha sendo reportada como mais efetiva que UVB NB no controle da psoríase, $, 4,6,9$ uma abordagem terapêutica padronizada para todos os casos de psoríase moderada a grave ainda não foi estabelecida. ${ }^{4,6}$

Como se sabe, a psoríase é uma doença de distribuição global que acomete ambos os sexos e ampla faixa etária.1,2 $\mathrm{Na}$ amostra estudada, de fato, ocorreu certa homogeneidade quanto à distribuição por sexo; ao mesmo tempo a idade dos pacientes tratados com fototerapia foi bastante ampla, partindo da segunda até a nona década de vida. Idade e sexo não são fatores limitantes na indicação de fototerapia. ${ }^{15}$
Em nossos serviços de fototerapia, UVB NB foi mais frequentemente indicada que PUVA ( $76 \%$ e $24 \%$ respectivamente) para tratamento de pacientes com psoríase. Foi observado que muitos pacientes com psoríase apresentavam outras comorbidades que contraindicaram o tratamento com PUVA. Pacientes com envolvimento hepático ou em uso de medicações, como as utilizadas no tratamento da hipertensão, diabetes ou ainda anti-inflamatórias, receberam indicação de UVB NB devido à maior segurança nesses casos, ${ }^{15,17}$ considerando que algumas drogas podem aumentar a sensibilidade individual aos raios UV. Esse foi o caso de $28 \%$ dos pacientes tratados em nossa amostra: em 19\% prescreveu-se UVB NB devido ao uso de drogas fotossensibilizantes e em 9\%, devido a doença hepática.

Para pacientes jovens, é indicada UVB NB por representar menor risco de induzir câncer de pele em longo prazo.6,18 No grupo estudado, $12 \%$ dos pacientes tiveram como primeira escolha UVB NB por apresentarem idade abaixo de 20 anos. Isso se torna importante quando se considera o risco de câncer como resultado da exposição cumulativa de radiação UV, associado à alta expectativa de vida desses pacientes.

Além desses aspectos, UVB NB é mais frequentemente indicada que PUVA devido à 


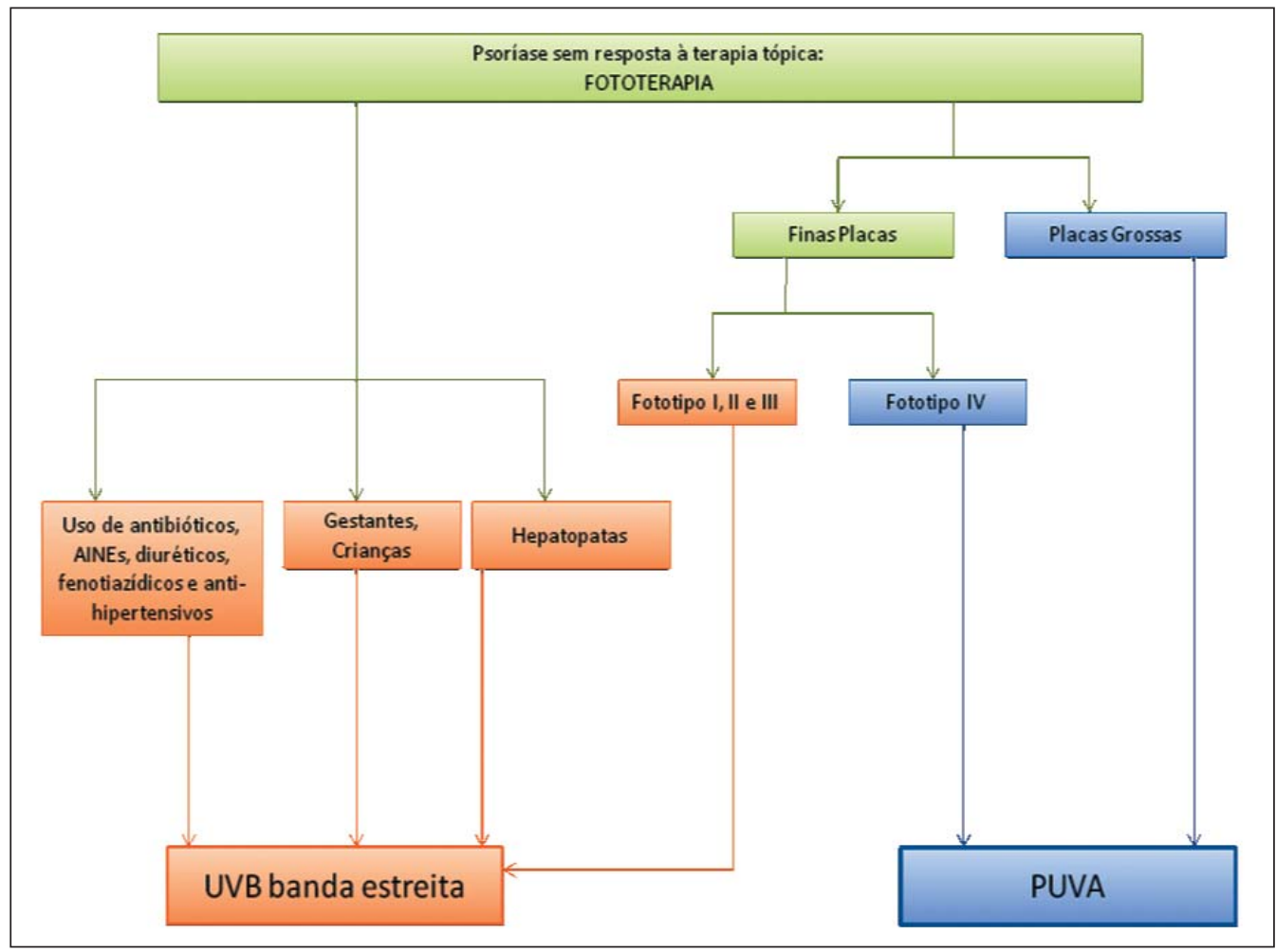

Figura 1: Indicação de fototerapia no tratamento de formas moderada $\mathrm{e}$ grave de psoríase: UVB de banda estreita (UVB NB) ou fotoquimioterapia PUVA praticidade de sua aplicação. ${ }^{6}$ A possibilidade de usar essa fototerapia na ausência de prescrição prévia de psoralênicos torna mais fácil a aceitação do tratamento por parte do paciente, uma vez que esses medicamentos muitas vezes trazem náuseas e outros efeitos colaterais. O uso de outras medicações pode ser mantido durante o tratamento com UVB NB. Entre os pacientes avaliados, PUVA foi mais indicada para pacientes com placas espessas e pele tipo IV a VI (classificação de Fitzpatrick).

Embora o objetivo deste estudo não tenha sido avaliar efetividade terapêutica, mas sim compreender como se comporta a prescrição da fototerapia na psoríase, foi possível observar que ambas as terapias foram efetivas. A média do PASI inicial nos pacientes tratados com PUVA foi de 14,9; no grupo tratado com UVB NB, foi de 10,4. Dentre os pacientes tratados com PUVA, o índice PASI 75 foi obtido em $75 \%$ dos casos, e bons resultados foram também alcançados em $80,4 \%$ dos pacientes tratados com UVB NB. A diferença entre os grupos não foi estatisticamente significante $(\mathrm{p}<0,05)$.

\section{CONCLUSÃO}

As prescrições de UVB NB excederam as de PUVA devido ao menor número de contraindicações e menor possibilidade de efeitos colaterais, e ainda por ser uma opção mais prática. Visto que tanto PUVA quanto UVB NB provaram ser efetivas no controle da psoríase, a opção por cada tratamento deve levar em conta a gravidade da doença, tipo de pele, uso de medicações e características do paciente. Uma avaliação clínica individualizada deve guiar a indicação entre um ou outro tipo de fototerapia. 


\section{REFERÊNCIAS}

1. Gudjonsson JE, Elder JT. Psoriasis: epidemiology. Clin Dermatol. 2007;25:535-46.

2. Schäfer T. Epidemiology of psoriasis. Review and the German perspective. Dermatology. 2006;212:327-37.

3. World Health Organization [homepage on the internet]. Are there beneficial effects of UV radiation? [cited 2008 Jun 18]. Available from: http://www.who.int/uv/faq/uvhealtfac/en/index1.html.

4. Naldi L, Griffiths CE. Traditional therapies in the management of moderate to severe chronic plaque psoriasis: an assessment of the benefits and risks. Br J Dermatol. 2005; 152:597-615.

5. Pearce DJ, Stealey KH, Balkrishnan R, Fleischer AB Jr, Feldman SR. Psoriasis treatment in the United States at the end of the 20th century. Int J Dermatol. 2006; 45 : 370-4.

6. Abel EA. Phototherapy: UVB and PUVA. Cutis. 1999;64:339-42.

7. Hönigsmann H. Phototherapy for psoriasis. Clin Exp Dermatol. 2001;26:343-50.

8. Coven TR, Walters IB, Cardinale I, Krueger JG. PUVAinduced lymphocyte apoptosis: mechanism of action in psoriasis. Photodermatol Photoimmunol Photomed. 1999; 15:22-7.

9. Barbagallo J, Spann CT, Tutrone WD, Weinberg JM. Narrowband UVB phototherapy for the treatment of psoriasis: a review and update. Cutis. 2001;68:345-7.

10. Walters IB, Burack LH, Coven TR, Gilleaudeau P, Krueger JG. Suberythemogenic narrow-band UVB is markedly more effective than conventional UVB in treatment of psoriasis vulgaris. J Am Acad Dermatol. 1999; 40(Pt 1):893-900.

11. Man I, Crombie IK, Dawe RS, Ibbotson SH, Ferguson J. The photocarcinogenic risk of narrowband UVB (TL-01) phototherapy: early follow-up data. Br J Dermatol. 2005; 152:755-7.

12. Diffey BL, Farr PM. The challenge of follow-up in narrowband ultraviolet B phototherapy. Br J Dermatol. 2007; 157:344-9.
13. Fitzpatrick TB. The validity and practicality of sunreactive skin types I through VI. Arch Dermatol. 1988;124:869-71.

14. Christophers E, Mrwietz U. Psoriasis. In: Freedberg IM, Eisen AZ, Wolff K, Austen KF, Goldsmith LA, Katz SI, editors. Fitzpatrick's Dermatology in General Medicine. New York: Mac Graw-Hill; 2003. p. 409-11.

15. Yones SS, Palmer RA, Garibaldinos TT, Hawk JL. Randomized double-blind trial of the treatment of chronic plaque psoriasis: efficacy of psoralen-UV-A therapy vs narrowband UV-B therapy. Arch Dermatol. 2006;142:836-42.

16. Sezer E, Erbil AH, Kurumlu Z, Tafltan HB, Etikan I. Comparison of the efficacy of local narrowband ultraviolet $\mathrm{B}$ (NB-UVB) phototherapy versus psoralen plus ultraviolet A (PUVA) paint for palmoplantar psoriasis. J Dermatol. 2007;34:435-40.

17. Ibbotson SH, Bilsland D, Cox NH, Dawe RS, Diffey B, Edwards C, et al. An update and guidance on narrowband ultraviolet B phototherapy: a British Photodermatology Group Workshop Report. Br J Dermatol. 2004;151:283-97.

18. Pasker-de Jong PC, Wielink G, van der Valk PG, van der Wilt GJ. Treatment with UV-B for psoriasis and nonmelanoma skin cancer: a systematic review of the literature. Arch Dermatol. 1999;135:834-40.

ENDEREÇO PARA CORRESPONDÊNCIA / MAILING ADDRESS:

\section{Ida Duarte}

Rua Monte Alegre, 523/101

05014000 São Paulo - SP

Tel./fax: 1138714018

E-mail: idaduarte@terra.com.br

Como citar este artigo/How to cite this article: Duarte I, Cunha JAJ, Bedrikow RB, Lazzarini R. Qual é o tipo de fototerapia mais comumente indicada no tratamento da psoríase? UVB banda estreita e PUVA: comportamento da prescrição. An Bras Dermatol. 2009;84(3):244-48. 